\title{
Intermédialités
}

Histoire et théorie des arts, des lettres et des techniques

Intermediality

History and Theory of the Arts, Literature and Technologies

\section{Mise en récit et mise en oeuvre. De l'enregistrement à la fiction dans les filatures de Sophie Calle}

\section{Perin Emel Yavuz}

Numéro 7, printemps 2006

Filer (Sophie Calle)

Shadowing (Sophie Calle)

URI : https://id.erudit.org/iderudit/1005518ar

DOI : https://doi.org/10.7202/1005518ar

Aller au sommaire du numéro

Éditeur(s)

Centre de recherche sur l'intermédialité

ISSN

1705-8546 (imprimé)

1920-3136 (numérique)

Découvrir la revue

Citer cet article

Yavuz, P. E. (2006). Mise en récit et mise en oeuvre. De l'enregistrement à la fiction dans les filatures de Sophie Calle. Intermédialités / Intermediality, (7), 89-109. https://doi.org/10.7202/1005518ar
Résumé de l'article

Dans le prolongement du Narrative art, Sophie Calle produit au tournant des années 1980 une oeuvre de photographies et de textes. Dans un rapport très étroit avec le réel, ses premiers travaux illustrent ce que Paul Ricoeur appelle la « configuration du temps en récit ». À partir du concept de la grille photographique, on mettra en évidence comment, dans Filatures parisiennes, Suite vénitienne et La filature, Sophie Calle fait basculer le récit factuel dans la fiction. 


\title{
Mise en récit et mise en œuvre
}

\author{
De l'enregistrement à la fiction \\ dans les filatures de Sophie Calle
}

\author{
Perin Emel Yavuz
}

\begin{abstract}
7 u début des années 1970 se développe une pratique photographique qui met en avant tout un aspect de l'œuvre d'art délaissé au profit de la forme depuis la fin du XIX ${ }^{\mathrm{e}}$ siècle, soit sa valeur narrative. De nombreux artistes de cette époque, de Duane Michals à ceux que la critique regroupera sous la bannière du Narrative art ${ }^{l}$ - parmi lesquels on retrouve Jean Le Gac, Christian Boltanski, Didier Bay pour les Français - élaborent ainsi des séquences photographiques plus ou moins courtes et complexes auxquelles ils associent du texte. Cet usage de la photographie permet de rompre avec un art devenu autonome, autoréflexif et enfermé dans le formalisme, dont le rapport au temps et au réel avait été quelque peu mis de côté. Si la notion de séquence autorise la réintroduction du temps - et par là même de la narration -, la photographie engage un rapport de l'œuvre au réel. Or, celui-ci, dans le cas du Narrative art, prend un caractère résolument subjectif, basé sur l'expérience quotidienne. Quelques années plus tard, alors que la période d'activité du Narrative art touche à sa fin, Sophie Calle commence sa production d'œuvres de photographies et de textes que l'on ne peut dissocier de ces expériences du début de la décennie, bien qu’elle soutienne qu'elle ne connaissait pas les travaux de ces artistes lorsqu'elle a commencé son propre travail ${ }^{2}$.
\end{abstract}

1. La période d'activité du Narrative art se situe entre 1973 et 1979, dont voici les plus importantes expositions: Story, John Gibson Gallery, New York, 1973; Narrative Art II, John Gibson Gallery, New York, 1974; Narrative Art, Palais des Beaux-arts, Bruxelles, 1974; Narrative Art, Cannaviello Studio d'Arte, Rome, 1974; Narrative Art 2, Cannaviello Studio d'Arte, Rome, 1975; American Narrative Art 1967-77, Contemporary Art Museum, Houston, 1978.

2. Anecdote relatée dans «Interview-biographie de Sophie Calle», dans Sophie Calle, M'as-tu vue, catalogue de l'exposition, Christine Macel (éd.), Paris, Éditions du Centre Pompidou, Éditions Xavier Barral, 2003, p. 79. 
Et pourtant, son œuvre, par l'association des deux médiums, écriture et photographie, par sa double nature narrative et hautement subjective, semble vraiment redevable à cette génération. Une différence majeure les sépare cependant: l'intention. Alors que les artistes du Narrative art, pour la plupart peintres de formation, interrogeaient leur statut d'artiste dans le contexte d'une prétendue mort de la peinture, les débuts de Sophie Calle se caractérisent par une absence de questions liées à la légitimité artistique de sa pratique et même de sa valeur, autorisée par l'éclatement des catégories et la diversification des pratiques - traits d'un postmodernisme déjà établi et reconnu. L'étude de ses premières filatures permettra, à travers la question de la mise en récit du réel par la photographie et le texte, de mettre en évidence la mise en oeuvre d'une pratique. Comme notre propos porte essentiellement sur deux formes - la série et la séquence photographiques - , nous commencerons par situer le cadre théorique dans lequel nous nous plaçons à partir d'une autre forme : la grille moderniste.

\section{DE LA GRILLE MODERNISTE À LA SÉQUENCE PHOTOGRAPHIQUE}

Dans un article de 1979 intitulé "Grids", analysant la peinture moderniste, Rosalind Krauss relève la constance d'une forme, la grille. Théorisant la récurrence de cette configuration dans la peinture du $\mathrm{xx}^{\mathrm{e}}$ siècle de Piet Mondrian à Ad Reinhardt en passant par Kasimir Malevitch et Agnes Martin, elle en fait «l'emblème de l'ambition moderniste4 » et le « paradigme ou [l]e modèle à l'anti-développement, à l'anti-récit, à l'anti-histoire5 ». La grille apparaît ainsi, selon l'auteur, comme la forme à travers laquelle il est possible d'identifier et de caractériser l'«ambition moderniste». Par sa nature rigide et répétitive, elle fige les formes et leur histoire puisqu'elle leur refuse toute évolution et, par là même, toute narration. Elle soustrait la peinture à la domination du réel et de sa représentation en la vouant à l'autoréflexivité. Ainsi, pour reprendre les mots de Didier Ottinger, la grille «incarne le fantasme d'un temps figé, elle est la gardienne vigilante de l’intégrité du modernisme pictural ${ }^{6}$. » Or, dès le début des années 1970, différen-

3. Rosalind Krauss, "Grids», October, n9, été 1979, p. 50-64. Repris et traduit sous le titre «Grilles", dans Rosalind Krauss, L'originalité de l'avant-garde et autres mythes modernistes, trad. Jean-Pierre Criqui, Paris, Macula, 1993, p. 93-110. Nous nous référerons à la traduction.

4. Rosalind Krauss, «Grilles », p. 93.

5. Rosalind Krauss, «Grilles», p. 108.

6. Didier Ottinger, "Spirales», Cahiers du Musée national d'art moderne, n $5^{8}$, hiver 1996, p. 131. 
tes stratégies artistiques remettent en cause ce refus de narration du modernisme. L'usage de la spirale, que pointe Didier Ottinger, utilisée par Robert Smithson, Bruce Nauman et Mario Merz, notamment comme une réplique à l'immobilité de la grille, incarnerait un aspect du refus de l'anti-naturalisme par sa nature de «forme ouverte, en expansion7». Métaphore de la croissance et du développement, elle réinstaure la notion de temps au sein de l'œuvre d'art. Le détournement ou la réappropriation de la forme de la grille par un autre médium apparaît comme une autre possibilité de briser le mutisme moderniste. Il s'agit de la photographie et de son usage séquentiel. Dès les années 1960 et plus encore dans les années 1970, des artistes utilisent la photographie en séquence, c'est-à-dire en «[...] une suite ordonnée de photographies qui possède une certaine cohé$\operatorname{sion}^{8}$.» Or, par analogie formelle, on peut identifier la forme de la grille dans la séquence photographique. Cependant, cette fois-ci, les cases - vides dans la grille moderniste - sont occupées par des photographies. Rassemblés et mis ainsi en relation, ces extraits du continuum, selon la théorie de l'enregistrement, rétablissent le temps et le mouvement au sein de la grille, ouvrant à nouveau cette grille - et par-là même l'œuvre d'art - à la représentation et à la narration.

Déployés sur le mur blanc de la salle d'exposition, les livres-installations de Sophie Calle, comme nous pourrions les nommer, revêtent eux aussi cette forme particulière de la grille séquentielle (fig. 1). C'est donc au sens le plus large que nous entendons le mot grille: l'agencement de cases remplies de photographies et de textes en un quadrillage rectiligne, l'agencement irrégulier de ces cases sur la surface du mur, ou encore la frise. Selon la théorie indicielle ${ }^{9}$, la photographie

7. Didier Ottinger, «Spirales », p. 132.

8. Danièle Méaux, La photographie et le temps. Le déroulement temporel dans l'image photographique, Aix-en-Provence, Publications de l'Université de Provence, 1997, p. 75.

9. La théorie indicielle repose sur la trichotomie indice-icône-symbole de Charles Sanders Peirce et pose la photographie comme une empreinte du réel. Partant de là, quelques théoriciens ont tenté, de la fin des années 1960 au début des années 1990, de définir l'ontologie du médium dans ses diverses applications. Parmi les textes les plus redevables à cette théorie, on peut compter ceux de Roland Barthes («Le message photographique », Communications, n ${ }^{\circ}$ 1, Paris, Éditions du Seuil, 1961, p. 128-130; « Rhétorique de l'image », Communications, n 4, Paris, Éditions du Seuil, 1964, p. 40-51; La chambre claire. Note sur la photographie, Paris, Cahiers du cinéma, Éditions Gallimard, Éditions du Seuil, 1980), de Rosalind Krauss (en particulier « Notes sur l'index. L'art des années 1970 aux États-Unis », Macula, nos 5-6, 1979, p. 165-175), de Jean-Marie Schaeffer (L'image précaire. Du dispositif photographique, Paris, Éditions du Seuil, 1987) et de Philippe Dubois (Lacte photographique et autres essais, Paris, Éditions Nathan, 1990). 

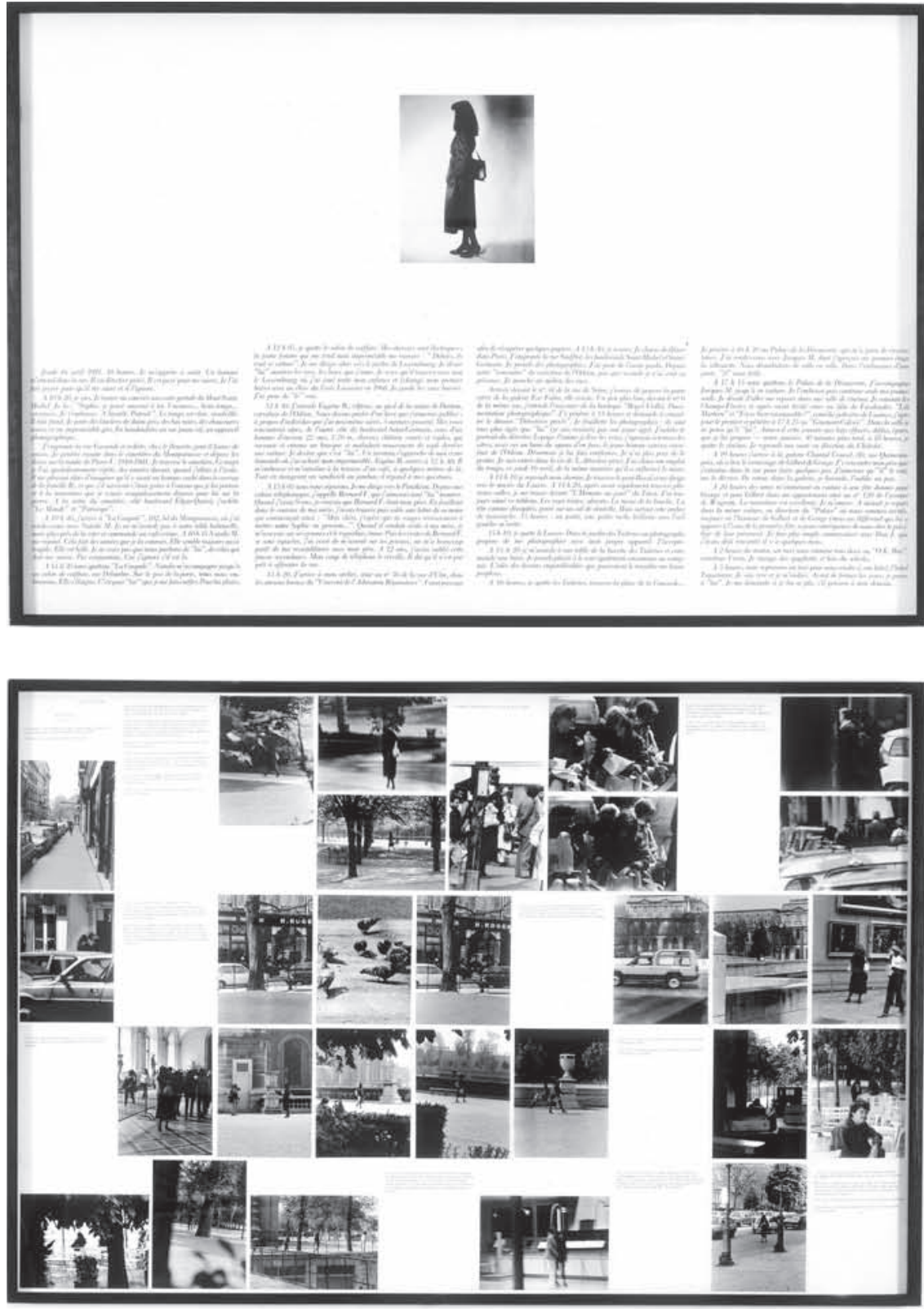

Fig. 1. Sophie Calle, La filature, vue d'installation, Galerie Emmanuel Perrotin, Paris, 1981-2001. (C) Sophie Calle/SODRAC 2006. Gracieuseté de la Galerie Emmanuel Perrotin, Paris/Miami. 
apparaît comme une «coupe » spatiale et temporelle ${ }^{1 \circ}$, un extrait figé du réel. Si la faculté narrative d'une image fixe reste au cœur des interrogations, une série d'images fixes réunies peut produire, de fait, par leur mise en relation, un cheminement narratif. Relevant d'abord de la série, en ce qu'elles impliquent une succession de clichés sans recherche de construction temporelle, elles assurent un passage vers la séquentialité. Tout en posant les fondations de son travail à venir, les premières œuvres de Sophie Calle ouvrent progressivement la voie à la mise en récit du monde qui l'entoure et de sa vie. Ainsi, les Filatures parisiennes, la Suite vénitienne et La filature" ${ }^{11}$ par leur forme et leur thème, apparaissent comme le lieu où se produit cette transformation du temps en récit, passage du réel informe au récit construit, mais aussi celui du devenir-artiste de Sophie Calle jusqu'à la naissance de son propre personnage.

\section{CONTENANCE}

La nature indicielle a donné à la photographie, dans l'un de ses usages, une valeur documentaire. Largement utilisée dans les sciences, elle participe aux activités d'identification, d'observation, de recensement et de classement, puis s'insère dans des répertoires préparés à les recevoir. Comme l'écrit John Elderfield dans l'article «Grids ${ }^{12}$ », la grille a une fonction « contenante » qui a intéressé les artistes - parmi lesquels Torres-Garcia ou Gottlieb, et leurs pictographes - mais qui reste mineure et dédaignée en peinture. La notion de «contenance » (containment, dans le texte) induit une mise en rapport des éléments des différentes cases entre eux. Ce système corrélatif provoque alors une historicisation des contenus de la grille, un va-et-vient aux conséquences narratives. Cependant, dans ce cas, la grille fonctionne comme une structure consciente. Elle morcelle et distribue volontairement l'espace et les objets qu'elle contient. Elle construit d'abord pour ensuite être emplie.

10. Voir Philippe Dubois, «Le coup de la coupe. La question de l'espace et du temps dans l'acte photographique», dans L'acte photographique, p. 151-202.

11. Ces trois travaux ont été regroupés et édités dans Sophie Calle, À suivre... (Préambule, Suite vénitienne, La filature), Doubles-jeux (livre IV), Arles, Actes Sud, 1998. Les titres sont inchangés sauf les Filatures parisiennes qui apparaissent sous le titre de «Préambule». Suite vénitienne a été publiée une première fois aux Éditions de l'Étoile, Paris, en 1983. Désormais, les références à l'ouvrage À suivre... seront indiquées par le sigle «AS » suivi de la page et placées entre parenthèses dans le corps du texte.

12. John Elderfield, «Grids», Artforum, vol. 10, nº 9, mai 1972, p. 55. 


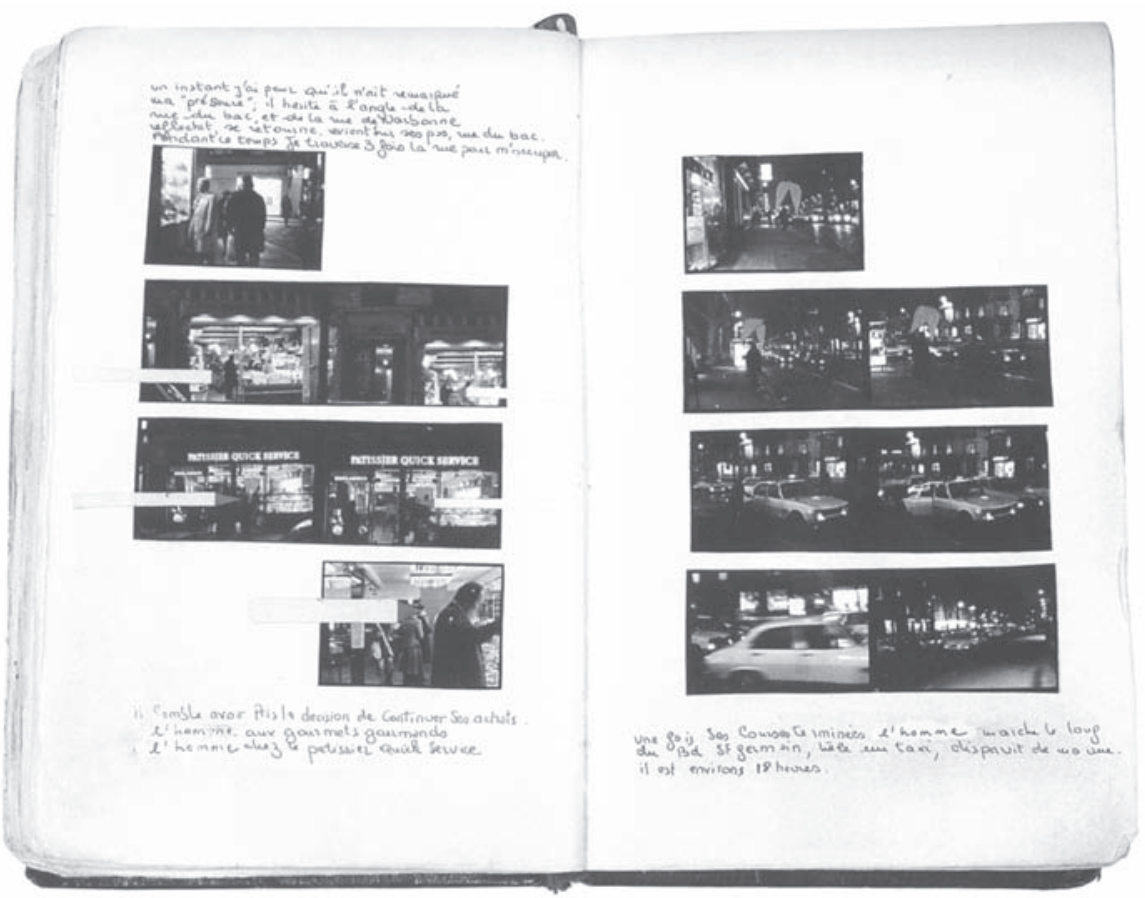

Fig. 2. Page du 21 janvier. Sophie Calle, « Préambule », dans À suivre..., Doubles-jeux (livre IV), Actes Sud, 1998. (C) Sophie Calle/SODRAC 2006. (C) Actes Sud 1998.

Les Filatures parisiennes répondent à ce schéma de la grille comme pur contenant de moments du réel. Dans cette série de cahiers, Sophie Calle répertorie les succincts comptes rendus de photographies et de textes des filatures d'inconnus qu'elle s'impose (fig. 2). Définissant son entreprise, elle écrit: «Je suivais des inconnus dans la rue.» (AS, «Préambule», p. 11) Le lundi $1^{\text {er }}$ janvier 1979, elle en précise même la périodicité: «Je prends de bonnes résolutions. Tous les jours je suivrai quelqu'un.» (AS, p. 19) Chaque filature est ainsi déclenchée par un détail dont la nature est tout aussi variable que subjective: "parce que sa coiffure m'avait étonnée » (AS, p. 13), «parce qu'elle croulait sous le poids d'un paquet» (AS, p. 13), «parce qu'elle se retourne sans cesse». (AS, p. 15) Si un élément déclencheur provoque chaque filature, l'ensemble de cette entreprise n'est motivé par rien de particulier. Elle évoque rapidement le plaisir que lui procure cette activité avant d'écrire de façon très laconique: «Je suis pour suivre.» (AS, p. 16) Bien que cette entreprise soit sans but, Sophie Calle prend pourtant soin de la subordonner à des règles comme celle de l'anonymat: «Je ne suivrai que des inconnus, dont je découvrirai peut-être certaines de leurs habitudes mais 
qui doivent rester des inconnus. » (AS, p. 16) Une seule exception se glisse dans ce catalogue de filatures, il s'agit de celle de Denis Roche, comme une sorte de signe annonciateur de la Suite vénitienne. Sans aucun dessein, ces filatures n'ont finalement d'autre sanction que l'«oubli³ », c'est-à-dire qu'elle ne leur donne pas suite.

Cette série de filatures apparaît ainsi comme une prise de conscience du monde sans toutefois pouvoir lui donner un sens. Activité de «plaisir » avant tout, elle revêt la forme d'un jeu sans but mais avec des règles. Mais le jeu n'est-il pas justement un moyen d'appréhender le monde et d'éveiller sa conscience à celuici? La grille que nous avons décrite n'a alors d'autre finalité que l'enregistrement des différents points de la filature mais est incapable de formuler un récit. La grille textuelle de l'emploi du temps s'accompagne de coupes immobiles d'une durée, séries de clichés chacune unie par la filature qui la provoque. Cependant, ce premier volet reste dans le domaine de la pure restitution. Il constitue une «pré-compréhension du monde de l'action ${ }^{14}$ » dans le sens que donne Paul Ricœur à mimésis I dans Temps et récit:

[...] imiter ou représenter l'action, c'est d'abord pré-comprendre ce qu'il en est de l'agir humain: de sa sémantique, de sa symbolique, de sa temporalité. C'est sur cette précompréhension, commune au poète et à son lecteur, que s'enlève la mise en intrigue et, avec elle, la mimétique textuelle et littéraire.

Il est vrai que, sous le régime de l'œuvre littéraire, cette pré-compréhension du monde de l'action recule au rang de « répertoire» [...], ou au rang de «mention » [...]. Il reste qu'en dépit de la coupure qu'elle institue, la littérature serait à jamais incompréhensible si elle ne venait configurer ce qui, dans l'action humaine, fait déjà figure ${ }^{15}$.

Ainsi, ces cahiers prennent l'allure d'une grille sérielle d'observations qui « figure» mais ne configure pas encore. Le récit ne peut encore émerger de cette double restitution scripturale et visuelle des faits parce que, sans trame, son degré de construction reste celui d'une succession - un « répertoire» - de moments. Ce « repérage » photographique et textuel qui donne des indications sur le temps et les choses n'est pas sans évoquer le principe du time-code inventé par Pierre Beauviala et dont l'artiste Philippe Parreno rappelle la définition dans son texte «Sitcom Ghost» :

13. Elle écrit à propos de ses suivis: «puis finalement les perdais de vue et les oubliais. » (AS, p. 11)

14. Paul Ricœur, Temps et récit. Tome 1. L'intrigue et le récit historique, Paris, Éditions du Seuil, coll. «Points Essais», 1983, p. 108.

15. Paul Ricœur, Temps et récit, p. 125. 
Le time-code est une méthode d'indexation électronique utilisée pour le montage et le chronométrage des séquences vidéo. Le time-code indique les heures, les minutes, les secondes et l'enchaînement des images sur une bande vidéo. C'est un système de symboles numériques ou analogiques servant à identifier des instants spécifiques. Le temps est utilisé pour préciser l'heure de la journée ou la mesure d'un intervalle de temps ${ }^{16}$.

Les Filatures parisiennes sont des moments bruts, indexés et mis bout à bout. Matière première du récit photographique, ce répertoire d'enregistrements en appelle désormais à une métamorphose du temps.

\section{GENÈSE}

Relatant ses débuts, Sophie Calle dit qu'elle ne se pensait pas artiste lorsqu'elle commença ses cahiers. C'est par le hasard d'une rencontre avec Bernard Lamarche-Vadel qu'elle le serait devenue. Celui-ci, intéressé par ses travaux, la désigna « $\operatorname{artiste}^{17}$ » ou tout du moins fit se précipiter les choses. Alors que les artistes de la génération précédente, dominés par le poids de l'histoire et du modernisme, interrogeaient leur statut d'artiste et par là même leur identité, Sophie Calle ne semblait pas se poser cette question. À la fin des années 1970, associer différents médiums, ancrer profondément son art dans le quotidien, faire de sa vie son art ne sont plus, comme ce fut le cas pour les artistes du début de la décennie, source de légitimations incessantes. De fait, Sophie Calle était une artiste, avant même de le savoir et c'est un regard, celui de Lamarche-Vadel, comme elle le raconte ${ }^{18}$, qui décela l'art que contenait sa vie. Voilà finalement comment se produisit le passage fortuit de ses petites occupations au statut d'activité artistique, par la hasard d'une présence qui devait l'identifier comme une artiste.

Par le même fruit du hasard, Suite vénitienne marque une avancée dans l'élaboration de l'œuvre. Cependant, cette fois-ci, le hasard ne se résume pas seulement à un constat qu'elle va accepter presque passivement. De la même façon que lorsqu'on la découvre artiste, le hasard apparaît comme ce qui va donner

16. Philippe Parreno, «Sitcom Ghost», dans Rirkrit Tirananija. Une rétrospective (Tomorrow is Another Fine Day), catalogue de l'exposition, Musée d'art moderne de la Ville de Paris/ARC, du 10 février au 20 mars 2005, p. 42.

17. «C'était en 1979. Bernard Lamarche-Vadel était le compagnon de la septième dormeuse. Elle lui raconta sa nuit. Il vint me voir et me proposa de montrer, au musée d'Art moderne de la Ville de Paris - à l'occasion de la Biennale des jeunes - , ce qui n'était pas encore un "projet artistique" mais plutôt un jeu. Ce fut ma première exposition. En fait, c'est lui qui décida que j'étais une artiste.» (Sophie Calle, Les dormeurs, Arles, Actes Sud, 200o, vol. 2, p. 7)

18. Sophie Calle, Les dormeurs, p. 7. 
forme. La qualifier d'artiste, c'est finalement dire que ce qu'elle fait est de l'art. C'est ainsi placer son activité dans un champ, lui donner sinon une finalité, tout du moins une valeur. Le hasard, dans Suite vénitienne, va, cette fois-ci, être non seulement le point de départ mais aussi - et surtout - le matériau fertile dont Sophie Calle va se servir activement pour réaliser cette œuvre. Elle écrit:

Àla fin du mois de janvier 1980, dans les rues de Paris, j’ai suivi un homme dont j’ai perdu la trace quelques minutes plus tard dans la foule. Le soir même, lors d'une réception, tout à fait par hasard, il me fut présenté. Je lui dis que je l'avais suivi dans l'après-midi et lui racontai pourquoi. Au cours de la conversation, il me fit part d'un projet imminent de voyage à Venise. Je décidai alors de m’attacher à ses pas, de le suivre. (As, p. 38)

Le hasard relève de l'informe. Suivre une personne choisie sans réelle motivation repose sur le hasard, un hasard de circonstance. Cela aurait pu tout aussi bien être une autre personne. Mais rencontrer cette personne dans une réception, le jour même, dans un lieu déterminé ne peut être considéré de façon anecdotique. Cette redondance du hasard apparaît comme la naissance, certes arbitraire, d'une forme, d'une mise en forme du réel, d'un événement. Et c'est à partir de cet événement arbitraire - élément déclencheur au sens fort - que Sophie Calle va justifier cette filature. Dans Temps et récit, Paul Ricœur écrit que la dimension configurante de la mise en intrigue consiste en la «transform[ation] des événements en histoire19. ${ }^{19}$ C'est ce que fait ici Sophie Calle: elle s'approprie cet événement de la rencontre pour nous raconter l'histoire de cette filature à Venise.

«Sans time-code, comment pourrions-nous transformer nos existences en récits $^{20}$ ? », interrogeait Philippe Parreno. Si le time-code relève de l'image en mouvement, il n'en révèle pas moins sa nécessité pour le montage, en l'occurrence l'organisation, la mise en forme d'images prises en temps réel afin de produire un récit. Et nous pourrions même remplacer le mot «time-code» par «série photographique» qui figure là le détachement d'un temps dans un autre, non sans évoquer la définition de Christian Metz, selon laquelle "[l]'une des fonctions du récit est de monnayer un temps dans un autre ${ }^{21}$.» Cela suppose donc que ce temps monnayé, ces «instants spécifiques» (pour reprendre les termes de la définition du time-code) soient choisis, extraits du temps réel et réagencés pour former un nouveau temps, celui de la narration. Ainsi, dès lors que Sophie Calle

19. Paul Ricœur, Temps et récit, p. 129.

20. Philippe Parreno, «Sitcom Ghost», p. 42.

21. Christian Metz, «Remarques pour une phénoménologie du narratif», dans Essais sur la signification au cinéma, tome 1, Paris, Klincksieck, 1968, p. 27. 
identifie la double rencontre de Henri B. comme un événement, elle va pouvoir dérouler le récit de sa filature à Venise.

Or, ici encore, la grille de photographies sérielles et de textes est entièrement redevable de l'enregistrement et du descriptif. Si Sophie Calle s'empare d'un événement comme point de départ à ce récit, celui-ci reste factuel. Elle rapporte heure par heure, jour après jour, l'évolution de cette filature, le tout documenté de photographies. L'excuse qu'elle donne à un passant selon laquelle elle attend un homme dont elle serait amoureuse ${ }^{22}$ n'a d'autre motivation que d'obtenir des informations sur ce qu'il fait et non de donner sens à ce récit. Si elle abandonne parfois la filature pour quelques rencontres, celles-ci restent dans le registre de l'anecdotique et ne participent pas au développement de l'intrigue, qui avance péniblement d'ailleurs. Et même lorsqu'elle est enfin démasquée par Henri B., il ne se passe rien. Aucune transformation ne se produit à partir de la rencontre entre un état $e_{1}$ et un état $e_{2}$, entre l'épisode de la recherche et l'épisode qui suit la rencontre. Aucune résolution non plus, comme elle le constate:

Qu'avais-je imaginé? qu'il allait m’emmener, me provoquer, m’utiliser? Henri B. n’a rien fait, je n'ai rien découvert. Il fallait une fin banale pour cette histoire banale ${ }^{23}$.

Cette filature reste ainsi à l'état d'enquête, une recherche d'éléments pour parachever la transformation de l'événement de la première rencontre en histoire. Cependant, lorsque Henri B. la photographie au moment où il la démasque, il signifie par ce geste que l'objet de l'enquête n'est autre qu'elle. Et c'est certainement pour cela qu'il refuse d'être photographié de face à ce moment-là. Son visage doit rester invisible parce qu'il apparaîtrait comme la résolution de l'enquête. Or, la Suite vénitienne n'est pas l'histoire d'une enquête mais celle d'un devenir. Jean Baudrillard n'écrit-il pas dans «Please follow me»: «Le réseau de l'autre est utilisé comme façon de vous absenter de vous-même. Vous n'existez que dans la trace de l'autre, mais à son insu, en fait vous suivez votre propre trace, presque à votre insu ${ }^{24} » ?$

C'est d'ailleurs après cette dernière rencontre avec Henri B. qu'elle tourne l'objectif vers elle dans son déguisement vénitien de «suiveuse » (pardessus beige, perruque blonde et chapeau). La transformation dans la Suite vénitienne réside alors dans la prise de conscience qu'elle en est elle-même l'objet (figs. 3-4).

22. «Je lui dis que j’aime un homme - seul l'amour me semble avouable - qui se trouve depuis 18 h 15 chez l'antiquaire Luigi, en compagnie d'une femme.» (Sophie Calle, Suite vénitienne, Paris, Éditions de l'Étoile, 1983, p. 44)

23. Sophie Calle, Suite vénitienne, p. 57.

24. Jean Baudrillard, «Please follow me», dans Sophie Calle, Suite vénitienne, p. 82. 


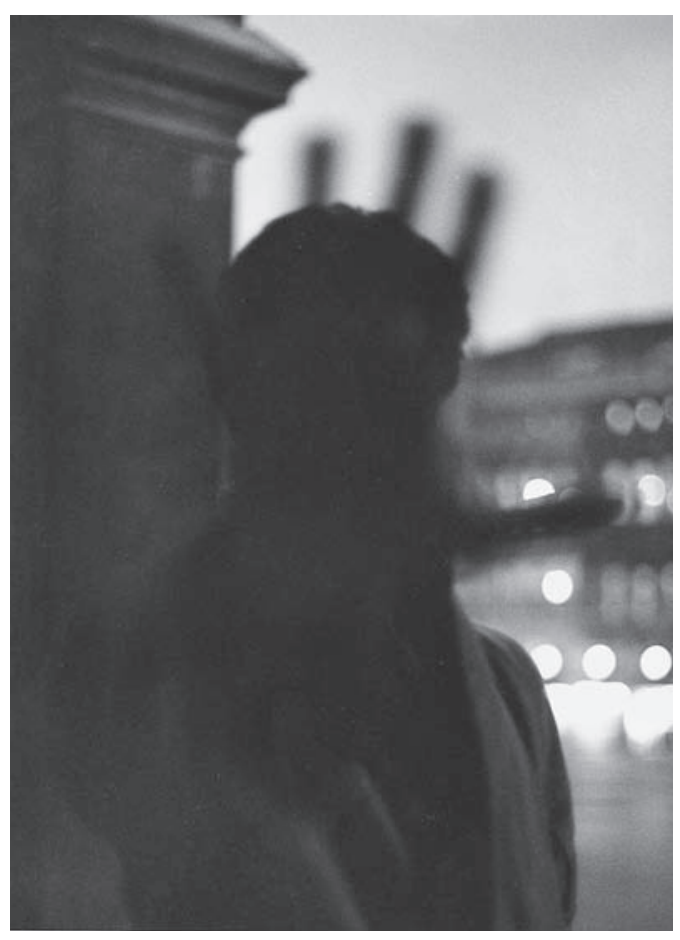

Fig. 3. Sophie Calle, « Suite vénitienne », dans À suivre..., Doubles-jeux (livre IV), Actes Sud, 1998. (C) Sophie Calle/SODRAC 2006. (c) Actes Sud 1998. 


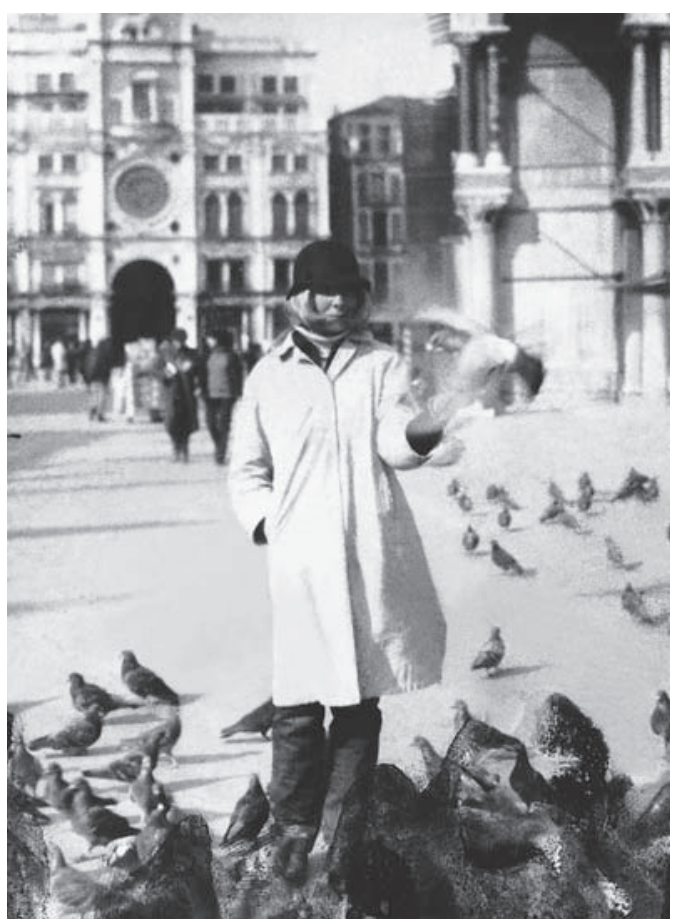

Fig. 4. Sophie Calle, "Suite vénitienne », dans À suivre..., Doubles-jeux (livre IV), Actes Sud, 1998. (C) Sophie Calle/SODRAC 2006. (C) Actes Sud 1998. 


\section{NAISSANCE}

La filature marque une nouvelle étape dans l'élaboration de l'œuvre de Sophie Calle. Alors que Suite vénitienne adhérait encore au réel et tentait finalement de le restituer assez fidèlement, le désir de le contrôler - par la mise en scène notamment - caractérise cette nouvelle œuvre. Le point de départ n'est plus un événement que le hasard a mis sur le chemin de Sophie Calle. C'est elle-même qui le provoque et le construit par un dispositif descriptif d'où émerge, en filigrane dans la grille à nouveau contenante, la fiction. Sophie Calle expose ainsi le scénario de La filature:

Selon mes instructions, dans le courant du mois d'avril 1981, ma mère s'est rendue à l'agence «Duluc détectives privés». Elle a demandé qu'on me prenne en filature et réclamé un compte rendu écrit de mon emploi du temps ainsi qu'une série de photographies à titre de preuve. («La filature», AS, p. 111)

Et pour s'assurer de la réussite de sa création, elle demande à un ami témoin de photographier le dispositif de la filature et d'en faire à son tour un compte rendu. Ainsi, Sophie Calle construit au travers de la grille qui devient dès lors triple (la sienne, celle du détective et celle de l'ami témoin), un enchevêtrement et une stratification des niveaux de la narration et de l'énonciation par l'improbable filature d'elle-même. Celle-ci, malgré son incongruité, signe la prise de contrôle de sa création. Si la photographie semble entretenir un rapport étroit avec le récit autobiographique par sa nature indicielle, son immédiateté présupposée peut néanmoins être remise en cause. Sophie Calle instaure effectivement, au préalable, un dispositif dans lequel elle distribue les rôles : il y aura la suivie, le suiveur et le témoin. Le hasard qui semblait décider de son entreprise n'a dorénavant qu'une infime place. Le rôle de la grille iconico-textuelle ne sera plus d'extraire - en lui donnant forme - un temps dans un autre mais d'attester de ce contrôle même si elle n'a pu maîtriser totalement le détective parfois ennuyé par l'inintérêt de la journée de l'artiste.

Cependant, la valeur narrative de cette ouvre semble être mise en doute par ce triple dispositif. Ce sont bien trois comptes rendus d'activité juxtaposés les uns aux autres qui la composent et qui convergent tous sur Sophie Calle (figs. 5 à 7 ). Suite vénitienne assurait l'émergence d'une intrigue par la filature de Henri B. même si elle ne reposait que sur un dénouement déceptif. Chaque grille de La filature ne contient en elle aucune intrigue mais uniquement des faits. Et pourtant, le dispositif instaure une dimension fictionnelle soupçonnée déjà dans Suite vénitienne. Tout d'abord, le rapport texte-image dans une entreprise à tendance autobiographique induit une distanciation entre l'auteur et lui-même, à l'image 
$A_{\text {I4 }} h$ 20, après avoir rapidement traversé plusieurs salles, je me trouve devant L'Homme au gant du Titien. J'ai toujours aimé ce tableau. Les yeux tristes, absents. La moue de la bouche. La tête comme décapitée, posée sur un col de dentelle. Mais surtout cette ombre de moustache. 15 heures : un point, une petite tache brillante sous l'œil gauche m'irrite.

Is h Io, je quitte le Louvre. Dans le jardin des Tuileries, un photographe propose de me photographier avec mon propre appareil. J'accepte.

A Is h 20, je m'assieds à une table de la buvette des Tuileries et commande une bière. Je prends plaisir à le voir également consommer au comptoir. L'idée des besoins impondérables qui pourraient le tenailler me laisse perplexe.

A I6 heures, je quitte les Tuileries, traverse la place de la Concorde. Je pénètre à $16 \mathrm{~h} 30 \mathrm{au}$ Palais de la Découverte, qui m'a paru de circonstance. J'ai rendez-vous avec Jacques M. dont j'aperçois au premier étage la silhouette. Nous déambulons de salle en salle. Dans l'embrasure d'une porte, "il" nous frôle.

A I7 h IS, nous quittons le Palais de la Découverte. J'accompagne Jacques $M$. jusqu'à sa voiture. Je l'embrasse puis continue seule ma promenade. Je décide d'aller me reposer dans une salle de cinéma. Je remonte les ChampsElysées et après avoir hésité entre un film de

Fig. 5. Compte rendu de Sophie Calle (autour de 17 h 15). Sophie Calle, «La filature ", dans À suivre..., Doubles-jeux (livre IV), 1998. (C) Sophie Calle/SODRAC 2006. (c) Actes Sud 1998. 
Fassbinder, Lili Marleen, et Est-ce bien raisonnable?, comédie policière de Lautner, j'opte pour le premier et pénètre à 17 h 25 au Gaumont-Colisée. Dans la salle, je ne pense qu'à "lui". Aime-t-il cette journée aux buts effacés, diffus, épars, que je lui propose - notre journée?

Trente minutes plus tard, à I 8 heures, je quitte le cinéma. Je reprends ma route en direction du Châtelet.

A Ig heures, j'arrive à la galerie Chantal Crousel, 80, rue Quimcampoix, où a lieu le vernissage de Gilbert \& George. J'y rencontre mon père que j'entraîne dans la rue pour faire quelques pas. J'aimerais qu' "il" le voie, me le décrive. De retour dans la galerie, je bavarde, l'oublie un peu.

$A 20$ heures, des amis m'emmènent en voiture à une fête donnée pour George et pour Gilbert dans un appartement situé au n ${ }^{\circ} 120$ de l'avenue de Wagram. La nourriture est excellente. Je m'amuse. A minuit, je repars dans la même voiture, en direction du Palace où nous sommes invités, toujours en l'honneur de Gilbert et de George (mais un différend qui les a opposés à l'issue de la première fête a pour conséquence de nous ôter le privilège de leur présence). Je fais plus ample connaissance avec Dan J. que j'avais déjà rencontré il y a quelques mois. 


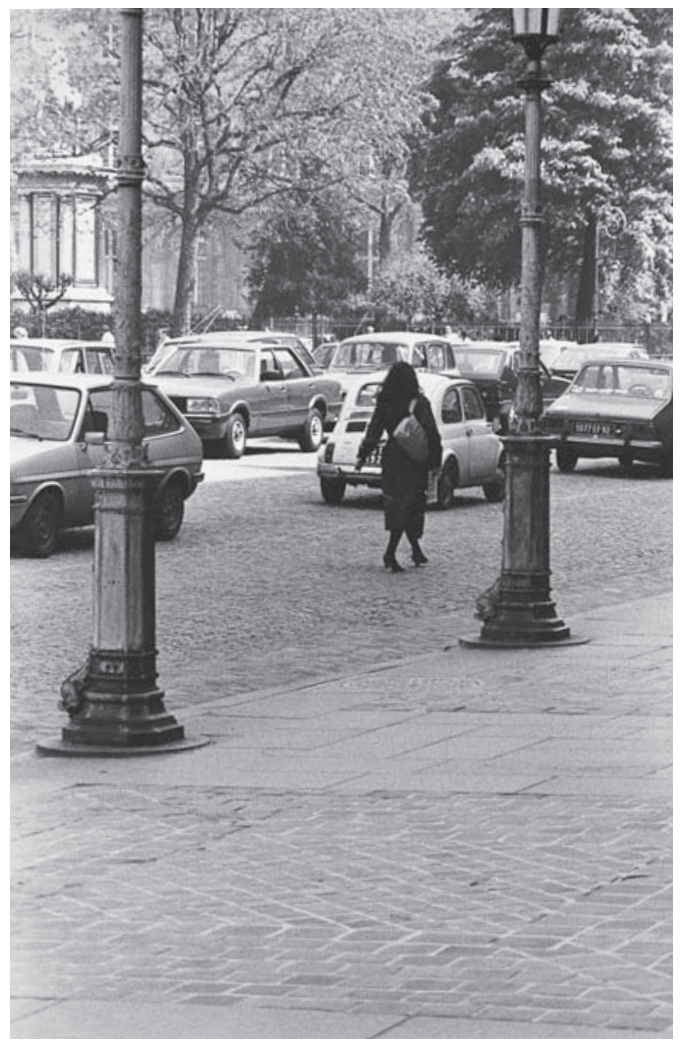

Fig. 6. Rapport du détective (à partir de 17 h 10). Sophie Calle, «La filature », dans À suivre..., Doubles-jeux (livre IV), Actes Sud, 1998. (C) Sophie Calle/SODRAC 2006. (c) Actes Sud $199^{8}$. 
A $17 \mathrm{~h} 10$, la surveillée et l'homme quittent le palais de la Découverte et se dirigent vers la rue Franklin-D. Roosevelt et se séparent à hauteur du $\mathrm{n}^{\circ} 1^{\prime}$ après s être embrassés. L' homme s'installe alors au volant d une Range Rover de couleur blanche immatriculée 383 BKX 75 et démarre.

A $17 \mathrm{~h} \mathrm{25,} \mathrm{la} \mathrm{surveillée} \mathrm{entre} \mathrm{au}$ cinéma Gaumont-Colisée 36, avenue des Champs-Elysées pour voir le film Lili Marleen.

A $19 \mathrm{~h} \mathrm{25,} \mathrm{la} \mathrm{surveillée} \mathrm{quitte}$ le cinéma et s'engage dans la station de métro Franklin-Roosevelt et monte dans une rame en direction du Pont-de-Sèvres. Elle change à Trocadéro, va en direction de Nation.

A 19 h 55, la surveillée descend à la station Denfert-Rochereau.

A 20 heures, la surveillée regagne son domicile. Nous levons la surveillance. 
Je voulais garder le souvenir de celui qui allait me suivre. Je ne savais pas quel jour de la semaine aurait lieu la filature. J'ai donc demandé à François M. de se poster chaque jour à 17 heures devant le Palais de la Découverte et de photographier quiconque semblerait me surveiller. Je lui ai recommandé la discrétion. J'ai reçu le rapport suivant, accompagné d'une série de photographies : "Ce jeudi 16 avril 1981, à 17 h 15 environ, Sophie Calle est sortie du Palais de la Découverte. J'ai immédiatement remarqué qu'un jeune homme d'environ 25 ans, vêtu d'un blouson de cuir, avec un appareil photographique autour du cou et une sacoche en bandoulière, la suivait. Il marchait à une vingtaine de mètres derrière elle et l'a photographiée au premier carrefour. J'ai mis la même distance entre lui et moi et je l'ai photographié à mon tour. Nous avons emprunté l'avenue Franklin-Roosevelt et traversé les Champs-Elysées. A 17 h 25, Sophie Calle est entrée au cinéma GaumontColisée. L'homme s'est attardé quelques instants ; il m'a semblé qu'il relevait les horaires des séances. Puis il a continué son chemin et il a remonté l'avenue jusqu'au Lord Byron qui avait à l'affiche Emmanuelle et les filles de Madame D. A 17 h 30, l'homme pénétrait dans le cinéma et disparaissait de ma vue."

Fig. 7. Rapport du témoin (de 17 h 15 à 17 h 30). Sophie Calle, «La filature ", dans À suivre..., Doubles-jeux (livre IV), Actes Sud, 1998. (c) Sophie Calle/SODRAC 2006. (c) Actes Sud 1998. 

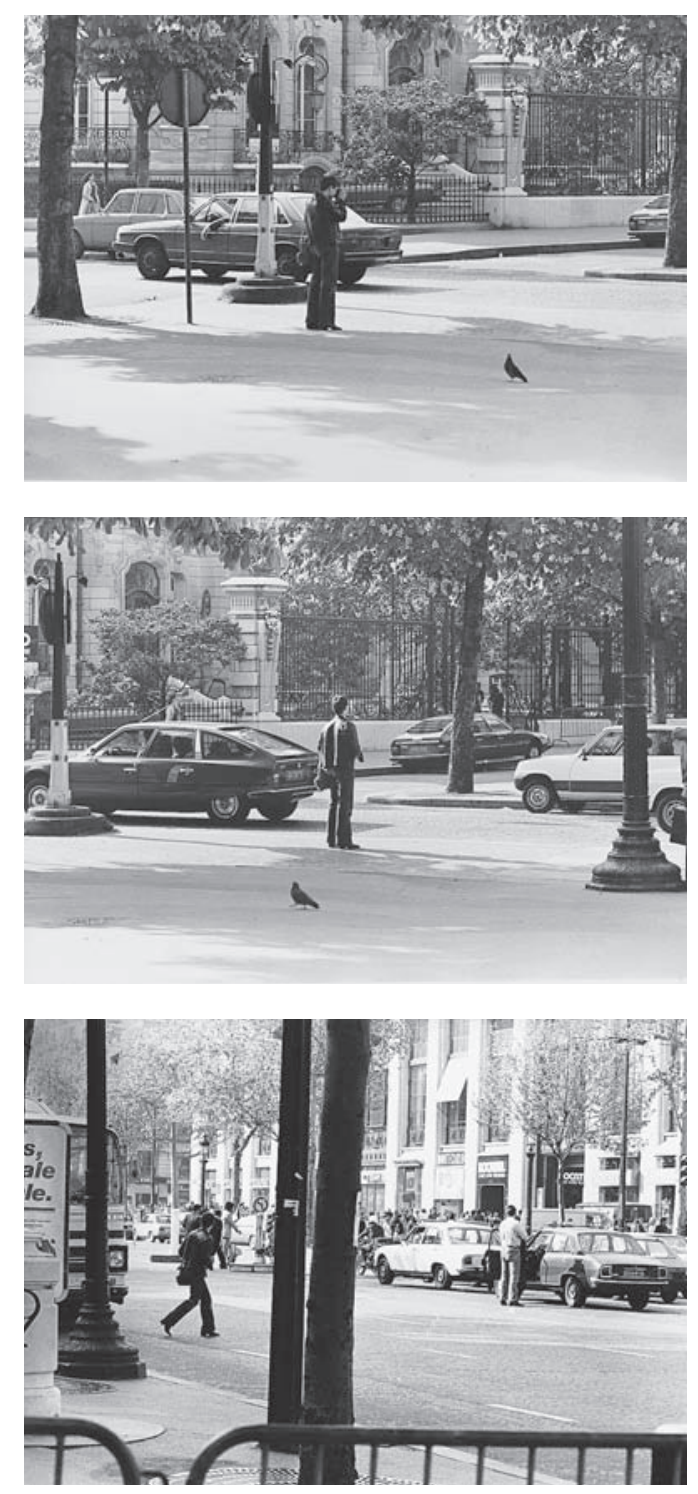
de l'écart entre l'instantané photographique et le temps de l'écriture. Comme le souligne François Soulages dans «Récit et photographie»:

[...] le photographe qui écrit «à côté » de ses photos a toujours un collaborateur, même si ce dernier est de fait lui-même: cette schize du photographe - celui qui photographie et celui qui écrit - semble fissurer et rendre impossible le récit autobiographique accompagné d'un texte, même si ce texte n'est qu'un titre ou qu'une signature - le nom propre et le corps propre étant, comme on le sait, fondateurs de toute autobiographie ${ }^{25}$.

Jusqu’ici, il était plus ou moins permis d’adhérer à ce désir de coller au plus près de la réalité exprimé par cette surprésence du «je» dans les parties textuelles et dans les restitutions photographiques («ce que je montre est ce que je vois », semble-t-elle nous dire). Or, la minutieuse précision avec laquelle Sophie Calle relate les faits apparaît comme surfaite. Cet excès atteint son paroxysme lorsque, dans Suite vénitienne, elle demande à un passant de la photographier à Venise, adhérant alors au plus près d'un «ça a été » barthésien. C'est pourtant à ce moment, comme il a été dit plus haut, que l'entreprise bascule clairement dans la fiction. La «schize du photographe» prend alors tout son sens. En écrivant les circonstances de la réalisation du portrait juste à côté de celui-ci, elle, qui était auteur et narrateur, devient dès lors personnage, ce que confirme La filature dans laquelle elle est à la fois sujet et objet. Paul Auster, qui s'était inspiré du travail de Sophie Calle pour créer le personnage de Maria, écrit ainsi à propos de cette œuvre transposée dans Léviathan:

Quand il lui avait remis son rapport à la fin de la semaine elle avait eu l'impression, en examinant les photographies d'elle-même et en lisant les chronologies exhaustives de ses faits et gestes, qu'elle était devenue quelqu'un d'inconnu, qu'elle s'était transformée en une créature imaginaire ${ }^{26}$.

Ainsi, par le biais d'une forme descriptive et quasiment objective, l'artiste crée son propre personnage dont La filature, par son dispositif très contrôlé, apparaît comme l'acte de naissance. La présence du témoin n’a ainsi d'autre raison que la nécessité d'assister à l'objectivation de Sophie Calle par le détective, et d'en fournir la preuve.

25. François Soulages, «Récit et photographie», dans Claude Amey (dir.), Le récit et les arts, Paris, L'Harmattan, coll. «Arts 8», 1998, p. 111.

26. Paul Auster, Léviathan, trad. Christine Le Bœuf, Arles, Actes Sud, 1993 [1992], p. 88 . 


\section{CONCLUSION}

Le thème de la filature dans le travail de Sophie Calle illustre le parcours d'une suiveuse qui, de poursuite en poursuite, s'affirme à la fois comme artiste, auteur, narrateur et personnage. À travers la figure de la grille photographique et textuelle s'élabore une œuvre dont la matière première, le réel, prend forme pour devenir histoire. De ce découpage du réel en microrécits qu’est la grille sérielle, Sophie Calle tisse une histoire dont les Filatures parisiennes, Suite vénitienne et La filature apparaissent comme les séquences. Répondant à la définition que donne Jean-Marie Schaeffer de l'acte de $\operatorname{raconter}^{27}$, Sophie Calle articule chacune de ces trois œuvres par un élément qui autorise l'élaboration d'une intrigue et qui amène sa progression. Le premier se situe ainsi dans la coïncidence déterminante de la rencontre avec Henri B. dans une soirée, après l'avoir filé dans la journée, et signe le passage de ce qui n'était qu'un jeu à un projet de nature artistique. En apprivoisant le hasard, Sophie Calle devient artiste. Le second lien logique n'est autre que son portrait à Venise. En retournant l'objectif vers elle, Sophie Calle devient son propre objet, ce que le dispositif de La filature révèle avec art. Ainsi, l'histoire qui nous est contée au long des filatures n'est autre que la naissance d'un personnage, étrange homonyme d'une artiste française. Et, dans cette construction narrative, la forme dominante bascule de la grille sérielle comme simple structure contenante d'instants, à la grille séquentielle comme unité d'un récit.

27. «Lacte de raconter est la forme spécifique que prend la description verbale lorsqu'elle a pour objet des transformations d'états de fait, c'est-à-dire lorsque les faits qu'elle dénote sont des séquences événementielles. Autrement dit, la spécificité de la narration par rapport aux autres descriptions verbales réside dans la spécificité des liens logiques qui réalisent l'intégration réciproque des propositions élémentaires grâce à des liens de consécutions [...] et de causalité.» (Jean-Marie Schaeffer, «Narration visuelle et interprétation », dans Jan Baetens, Mireille Ribière (dirs.), Time, Narration and the Fixed Image, Amsterdam, Atlanta, Rodopi, 2001, p. 13) 